\title{
BROADCAST INTERACTIVE PROOFS
}

\section{(Extended Abstract)}

\author{
Mike Burmester* \\ Dept. of Mathematics \\ RHBNC - University of London \\ Egham, Surrey TW20 OEX \\ U.K.
}

\author{
Yvo Desmedt ${ }^{\dagger}$ \\ Dept. of EE \& CS \\ P.O.Box 784 \\ WI 53201 Milwaukee \\ U.S.A.
}

\begin{abstract}
In this paper we extend the notion of (single-verifier) interactive zero-knowledge proofs to (multi-verifier) broadcast proofs. In our scheme the prover broadcasts messages to many verifiers simultaneously. We consider two cases: one for which the number of rounds of messages exchanged is unbounded (as a function of the length of the common input $x$ ), and one for which it is constant. Compared to repeated single-verifier proofs (one proof for each verifier), the saving in broadcast bits is of the order of the number of verifiers in the first case, provided there are enough verifiers. More precisely, if the number of verifiers exceeds $\log |x|$ then there is "practically" no extra cost in broadcast bits by further increasing the number of verifiers. In the second case the saving in the number of rounds is "practically" $|x| / \log |x|$. An added feature of broadcast proofs of the second type is that they are sabotage-free.

Our scheme makes use of a network which directs the messages of the verifiers to the prover. The universality of the scheme derives from the way in which the network handles collisions.
\end{abstract}

*Supported by SERC grant GR/F 57700.

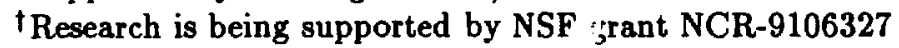




\section{Introduction}

Suppose that an authority (say the president of the U.S.A.) wants to prove something to a large number of verifiers. The proof can be related, for example, to a command or some action. One obvious solution would be to prove it separately to each verifier, sequentially. This solution however could be very time consuming, certainly when the number of verifiers is large. Another solution is to perform all the proofs in parallel. However in both cases the number of bits communicated could be enormous. Our solution is completely different. The prover will use a broadcast station and the verifiers will call in their questions. We shall take into account aspects such as the time it will take to run the protocol and the bandwidth, and the total number of bits that must be broadcast, which must be small. For us broadcasting is expensive and phone calls are cheap. Furthermore we shall use a network which reduces substantially the number of operators required to answer incoming calls.

When the same message is sent to many receivers broadcasting is the logical solution. Recently broadcast has been receiving a lot of attention in the research on cryptography. Fiat discussed the protection of privacy and selective broadcast [7] and Desmedt-Yung discussed the issue of authenticating broadcasted messages [6]. In this paper we discuss how broadcasting can simplify the prover's effort when multi verifiers want to check interactively a zero-knowledge proof.

In our model the prover is $P$ and the verifiers are $V_{1}, \ldots, V_{v} . P$ broadcasts to all the verifiers. Each $V_{i}$ has a one-directional line to a (telephone) network $W$. $P$ has $n$ incoming lines originating from $W$. These lines are "read-only". The prover $P$ communicates his messages to the $V_{i}$ by broadcasting them, whereas the $V_{i}$ will "phone-in" their "queries" through the network $W$. Each verifier selects a particular line of the prover. $W$ directs incoming calls to $P$ on a "first-come/first-serve" basis (and chooses randomly if there is a collision).

Our proof is a multi-verifier interactive zero-knowledge proof. Multiprover interactive proofs were introduced in [3] in a theoretical context. We are mainly concerned here with practical aspects, and our appr ach is completely different. Multi-language zero-knowledge proofs were recently dis- 
cussed in [12].

We remark that existing non-interactive zero-knowledge proofs, such as [4, 5], are not practical and many signature schemes are not zero-knowledge.

This note is organized as follows. In Section 2 we consider a formal setting for broadcast proofs. We recommend that the reader who is satisfied with the informal model above should omit this section in a first reading. In Section 3 we present a "sequential" broadcast zero-knowledge proof. In Section 4 we present a constant-round zero-knowledge broadcast proof. which is sabotagefree.

\section{A formal setting: definitions and nota- tions}

For a formal setting we use the framework in [9, pp. 4-10].

Definition 1 A Turing machine which is equipped with a read-only input tape, a work tape, a random tape, a history tape, $i$ read-only communication tapes and $j$ write-only communication tapes, is called an $(i, j)$-interactive Turing machine.

Observe that the interactive Turing machines in [9] have one read-only communication tape and one write-only communication tape. Below we use the abbreviations, ITM for an "interactive probabilistic Turing machine", and MITM for an $(i, j)$-interactive probabilistic Turing machine, when there is no ambiguity about $i$ and $j$.

Definition 2 A broadcast interactive protocol $\left(P, W, V_{1}, \ldots, V_{v}\right)$ consists of $v$ ITM's $V_{1}, \ldots, V_{v}$, one $(n, 1)$-ITM and one $(v, n)$-ITM, denoted by $P$ and $W$ respectively, which are such that:

- $P$ and $V_{1}, \ldots, V_{v}$ share a common communication tape which is writeonly for $P$ and read-only for all the $V_{i}$. When $P$ writes a string on this tape, we say that $P$ broadcasts that string.

- $P$ and $W$ share $n$ communication tapes which are read-only for $P$ and write-only for $W$. We call these tapes: incoming phone lines. 
- $W$ and $V_{1}, \ldots V_{v}$ share $v$ communication tapes which are read-only for $W$ and write-only for $V_{1}, \ldots, V_{v}$. We call these: outgoing phone lines.

$W$ is the network. Its purpose is to direct incoming calls from the $V_{i}$ to $P$ and to prevent "collisions": if more than one call is addressed to any particular line, then $W$ selects randomly which verifier receives the line.

Let $\left(P, W, V_{1}, \ldots, V_{v}\right)$ be a broadcast interactive protocol, $x$ be the input, and $\sigma$ be the concatenation of the strings written on the communication tapes by $P, W, V_{1}, \ldots, V_{v}$ and the random coin tosses of $V_{1}, \ldots, V_{v}$. We say that $\left(P, W, V_{1}, \ldots, V_{v}\right)$ outputs $\sigma$ on input $x . \quad\left(P, W, V_{1}, \ldots, V_{v}\right)(x)$ is the probability space which assigns to $\sigma$ the probability that $\left(P, W, V_{1}, \ldots, V_{v}\right)$ outputs $\sigma$ on input $x$. Joint-View $\left(P, W, V_{1}, \ldots, V_{v}\right)(x)$, is the joint probability space generated by $\left(P, W, V_{1}, \ldots, V_{v}\right)(x)$. Below we will use the notation $\tilde{P}$ or $\tilde{V}_{i}$ to indicate that $P$ or $V_{i}$ do not necessarily adhere to the program of $\left(P, W, V_{1}, \ldots, V_{v}\right)$. We use the notation $P, V_{i}$ only for participants who adhere to the program. We call such participants honest. We shall assume that the network $W$ is not corrupted. In other words, the anonymity of the callers is guaranteed. So a dishonest prover cannot single out a particular verifier.

Definition 3 Let $L \subset\{0,1\}^{*}$ be a language, $\left(P, W, V_{1}, \ldots, V_{v}\right)$ a broadcast interactive protocol, $x$ the common input. We say that $\left(P, W, V_{1}, \ldots, V_{v}\right)$ is a broadcast proof for $L$ if,

- Completeness (If $x \in L$ and if all the verifiers are honest then every verifier will accept the proof.) For any $c$, for all sufficiently large $x$, if $x \in L$ then $\forall i: \operatorname{Prob}\left(V_{i}\right.$ accepts $\left.x\right) \geq 1-|x|^{-c}$ (overwhelming).

- Soundness (If $x \notin L$ then an honest verifier will not accept.) For any $c$, for all sufficiently large $x$, for any dishonest proof system $\left(\tilde{P}, W, \tilde{V}_{1}, \ldots\right.$, $\left.\tilde{V}_{v}\right):$ if $x \notin L$ then for each honest $\tilde{V}_{j}: \operatorname{Prob}\left(\tilde{V}_{j}\right.$ accepts $\left.x\right) \leq|x|^{-c}$ (negligible).

Definition $4 \mathrm{~A}$ broadcast proof $\left(P, W, V_{1}, \ldots, V_{v}\right)$ for $L$ is zero-knowledge if it is possible to simulate the joint-view of the verifiers when $x \in L$. Formally, for any $x \in L$, for any ITM's $\tilde{V}_{1}, \ldots, \tilde{V}_{v}$ (possibly cheating), for 
any "histories" $\widetilde{H}_{1}, \ldots, \widetilde{H}_{v}$, there exist a probabilistic Turing machine $M$ running in expected polynomial time, such that the family of probability spaces $\left\{M\left(x ; \widetilde{H}_{1}, \ldots, \widetilde{H}_{v}\right)\right\}$ is indistinguishable from the family of probability spaces $\left\{\right.$ Joint-View $\left.\left(P, W, \widetilde{V}_{1}, \ldots, \widetilde{V}_{v}\right)\left(x ; \widetilde{H}_{1}, \ldots, \widetilde{H}_{v}\right)\right\}$. As in the case of single verifier proofs we have perfect, statistical, computational zero-knowledge proofs corresponding to the level of distinguishability [9].

Definition 5 Let $\left(P, W, V_{1}, \ldots, V_{v}\right)$ be broadcast proof for $L$. It is sabotagefree if

- For any $c$, for all sufficiently large $x$, for any dishonest proof system $\left(\widetilde{P}, W, \widetilde{V}_{1}, \ldots, \tilde{V}_{v}\right)$, with $\widetilde{P}=P:$ if $x \in L$ then for every honest $\tilde{V}_{j}$ : $\operatorname{Prob}\left(\tilde{V}_{j}\right.$ accepts $\left.x\right) \geq 1-|x|^{-c}$.

\section{Remarks}

(i) For us, $v$ and $n$ are functions of the input length $|x|$, and $v$ is polynomially bounded. Our formal model does not allow for this. In the final paper we will present a model which solves this problem.

(ii) One may wonder why we have introduced the sabotage-free aspect. Completenes implies that if $x \in L, V_{j}$ accepts $x$ only if all the other verifiers are honest. Because of the way our network is set up, it is not excluded that an honest prover will fail. When $x \in L$, dishonest verifiers could conspire against a honest verifier such that the last one will never accept.

(iii) Our model implicitly assumes that a dishonest verifier cannot eavesdrop on the questions asked by honest verifiers. To enforce this, privacy protection could be used when necessary.

(iv) We have avoided to use a model which is too restrictive. On the $n$ phone lines at the prover's premises many phone calls could be received before the prover answers, or the prover could just answer the first one, or he could wait a certain time and then answer when no further calls are received. Our model allows for this. 


\section{Broadcast proofs: A first approach}

\subsection{An introduction}

We first present a new "single-verifier" zero-knowledge proof on which we will build our scheme. This proof is based on concepts proposed by FiatShamir [8] and Guillou-Quisquater [10].

Let $a, b$, be constants, $0<a<b$. The common input is $x=(I, u, N)$, where $N$ be the product of large prime numbers, $I \in Z_{N}^{*}$, and $u$ is a prime with $|N|^{a} \leq u \leq|N|^{b}$, where $|N|$ is the binary length of $N$. Observe that $|N|=\Theta(|x|)$. The prover proves that there exists an $s$ such that: $I s^{u} \equiv$ $1(\bmod N)$. We call $s$ the "secret". Let $n=\left\lfloor\log _{2} u\right\rfloor$ and $s_{1}=s, s_{2}=s^{2}, \ldots$, $s_{n}=s^{2^{n-1}}, I_{1}=I, I_{2}=I^{2}, \ldots, I_{n}=I^{2^{n-1}}$. We assume that $P$ and $V$ have calculated all the $I_{j}$ from $I$ and $N$ before the protocol starts.

Observe that one generally assumes that it is hard to decide if a number $I$ has a $u$-th root modulo $N$ [1]. No correlation between the complexity of this problem and the choice for $u$ have been found so far. Therefore it is reasonable to restrict $u$ to be a prime with $|N|^{a} \leq u \leq|N|^{b}$. In the final paper we shall exhibit a protocol for a more general setting.

The protocol ${ }^{1}$ has as follows.

First $V$ verifies if $u$ is a prime and if $|N|^{a} \leq u \leq|N|^{b}$. Then, repeat $t=|x|$ times:

Step $1 P$ sends $V$ the number $z \equiv r^{u}(\bmod N)$ where $r \in_{R} Z_{N}^{*}$.

Step $2 V$ sends $P$ the bit string $\left(b_{1}, \ldots, b_{n}\right) \in_{R}\{0,1\}^{n}$ as a query.

Step $3 P$ halts the protocol if the query is not of the correct form; otherwise $P$ sends $V$ the number $y \equiv r \cdot \prod_{b_{j}=1} s_{j}(\bmod N)$.

Step 4 The verifier $V$ checks that $y \in Z_{N}^{*}$ and that $z \equiv y^{u} \cdot \prod_{b_{j}=1} I_{j}(\bmod N)$. If this is not so the protocol is halted.

If $V$ has not halted the protocol then $V$ accepts $x$.

\footnotetext{
${ }^{1}$ In the protocol, " $\in_{R}$ " indicates a random selection with uniform distribution, independently of earlier choices.
} 
Lemma 1 The protocol above defines a zero-knowledge proof of membership for the language $L=\left\{(I, u, N) \mid u\right.$ is a prime, $\left.|N|^{a} \leq u \leq|N|^{b}, I \in\left(Z_{N}^{*}\right)^{u}\right\}$.

Proof. The proof is straightforward and will be given in the final paper.

\subsection{A broadcast proof}

We will use the protocol above to describe a broadcast proof. The main difference is that we assign to each $s_{j}$ one incoming phone line. We assume that $P$ and all the $V_{i}$ have calculated the $I_{j}$ from $I$ and $N$ before the protocol starts. The network in this case operates, essentially, on a "first-come/firstserve" basis. However the caller does not know if his call has been accepted. The prover allows some time between broadcasts for the verifiers to make their calls. The broadcast protocol has as follows.

Repeat $T$ times:

Step $1 P$ sets $f_{i}^{J}=0$ for $i=0,1$ and $J=1, \ldots, n$. $P$ broadcasts the number $z \equiv r^{u}(\bmod N)$ where $r \in_{R} Z_{N}^{*}$.

Step 2 Each $V_{i}, i=1, \ldots, v$, sends: $W$ a line number $J_{i} \in_{R}\{1, \ldots, n\}$ and $P$ (via $W$ ) the query $b_{J_{i}} \in R\{0,1\}$. $P$ reads (simultaneously during this step) the incoming queries (scanning line after line). If on some line $J$, the incoming query $q \notin\{0,1\}$ then $P$ ignores it, else $f_{q}^{J}=f_{q}^{J}+1$.

Step $3 P$ broadcasts the number $y \equiv r \cdot \prod_{\bar{b}_{j}=1} s_{j}(\bmod N)$ where $\bar{b}_{j}$ have been randomly selected by $P$ such that $\operatorname{Prob}\left(\bar{b}_{j}\right.$ is selected $)=f_{\bar{b}_{j}}^{j} /\left(f_{0}^{j}+\right.$ $f_{1}^{j}$ ) and in case that both $f_{0}^{j}$ and $f_{1}^{j}$ are zero $\bar{b}_{j}$ is chosen randomly with uniform probability distribution.

Step 4 Each $V_{i}$ checks that $y \in Z_{N}^{*}$ and $z \equiv y^{u} \cdot I_{1}^{b_{1}} \cdots I_{J_{i}}^{b_{d_{i}}} \cdots I_{n}^{b_{n}}(\bmod N)$, by using his own $b_{J_{i}}$ and by allowing all possible values for the other queries $b_{j} \in\{0,1\}$ (this requires only $2^{n-1}<u \leq|N|^{b}=\Theta\left(|x|^{b}\right.$ ) operations). If one of these verifications is successful, then the verifier $V_{i}$ performs: success:=success +1 . 
Regardless of the outcome, the protocol is repeated for a maximum of $T$ times. At the end of the protocol a verifier will accept if and only if success $\geq t$, where $t=|x|$ is the number of iterations of the single verifier protocol.

\subsection{Theorem and proof}

Theorem 1 Suppose that:

(i) $n=O(\log |x|)$,

(ii) $d \geq n / v \geq b>0$, where $d=O(\log |N|)$ and $b=8 \cdot|x|^{-\frac{1}{4}}$.

(iii) $T=t /(p(1-c))$ is an integer, where $p=1 / 2+b\left(1-e^{-1}\right) / 2$ and $c$ is such that $c>t^{-\frac{1}{4}}$ and

$$
2 p(1-2 c)>1
$$

If the number of iterations of the broadcast protocol is (precisely) $T$, then $\left(P, W, V_{1}, \ldots, V_{v}\right)$ is a zero-knowledge broadcast proof for the language $L=$ $\left\{(I, u, N) \mid u\right.$ is a prime, $\left.|N|^{a} \leq u \leq|N|^{b}, I \in\left(Z_{N}^{*}\right)^{u}\right\}$.

\section{Remarks}

(i) A crude approximation for $T$ is $2 t$. So the honest prover can prove that $x \in L$ by "running twice" the single-verifier protocol, "irrespectively" of the number of verifiers.

(ii) Observe that for the broadcast proof of Section 3.2 each verifier asks only a one bit question, while in the single-verifier proof of Section 3.1 the verifier asks an $n$-bit question.

(iii) In the final paper we will generalize Theorem 1 . The main difference is that the queries $b_{i}$ of the verifiers $V_{i}$ are selected from a set $\{1, \ldots, q\}$, where $q \geq 2$.

Proof of Theorem 1. (It is suggested to skip this in a first reading.) We shall require the following lemmas: 
Lemma 2 If the prover $P$ is honest and if all the verifiers are honest, then the probability that the query of a verifier will be answered correctly is

$$
\bar{p}=\frac{1}{2}+\frac{n}{2 v}\left(1-\left(1-n^{-1}\right)^{v}\right) \text {. }
$$

Proof. Will be given in the full paper.

Lemma $3 \quad \bar{p} \geq p$.

Proof. Will be given in the full paper.

Lemma 4 Suppose that $x \notin L$. If the honest verifier $V_{i}$ asks the query $b_{i}$, then the probability $\tilde{p}$ that a (cheating) prover $\tilde{P}$ (any $\tilde{P}$ ) can answer this query correctly is no better than $1 / 2$.

Proof. Will be given in the full paper.

Lemma 5 We can choose the $c$ in (1) and in such a way that $c>t^{-\frac{1}{4}}$ and $T=t /(p(1-c))$ is an integer.

Proof. Will be given in the full paper.

We can now prove the main theorem.

Completeness (When all the verifiers are honest, the honest prover will answer correctly every verifier, for at least $t$ iterations out of $T$, with overwhelming probability.) We shall use Okamoto's bound [11, p. 69] for the tail probabilities of the binomial distribution. Let $\overline{\mathbf{z}}$ be the number of times in $T$ iterations that the honest prover $P$ will answer correctly the query of an honest verifier $V$. Then $\bar{p}$ is the expected value of $\overline{\mathbf{z}} / \mathbf{T}$ and,

$$
\operatorname{Prob}\{|\overline{\mathbf{z}} / T-\bar{p}| \geq \varepsilon\}<\exp \left[-2 T \varepsilon^{2}\right], \text { for any } \varepsilon>0 \text {. }
$$

We take $\varepsilon=c p$. By Lemma $5, T \varepsilon^{2}=t c^{2} p /(1-c) \geq w t^{\frac{1}{2}}, w$ a constant, since $p>1 / 2$. We are assuming that $t=|x|$, so $e^{-t}$ is negligible. Thus $\exp \left[-2 T \varepsilon^{2}\right]$ is negligible, and from (3), $\operatorname{Prob}\{\overline{\mathbf{z}} \leq \bar{p} T-\varepsilon T\}$ is negligible. By Lemma 5 , $t=p T(1-c)=p T-c p T=p T-\varepsilon T \leq \bar{p} T-\varepsilon T$, since $\bar{p} \geq p$ by Lemma 3 . So $\operatorname{Prob}\{\overline{\mathbf{z}} \leq t\}$ is negligible, and therefore $\operatorname{Prob}\{\overline{\mathbf{z}} \geq t\}$ is overwhelming. 
Soundness (The probability that a dishonest prover will answer correctly for $t$ or more iterations out of $T$ is negligible.) In this case $x \notin L$, but the prover $\widetilde{P}$ wants to make an honest verifier $V$ accept the proof. From Lemma 4 the probability $\tilde{p}$ that $\tilde{P}$ will succeed in having success $=1$ after the first round is at most $1 / 2$. If $\tilde{\mathbf{z}}$ is the number of times out of $T$ that $\tilde{P}$ will increase success then, as in the previous case, $\operatorname{Prob}\{\tilde{\mathbf{z}} / T-\tilde{p} \geq \varepsilon\}=\operatorname{Prob}\{\tilde{\mathbf{z}} \geq \tilde{p} T+\varepsilon T\}$ is negligible for $\varepsilon=c p$. Indeed, $\tilde{p} T+\varepsilon T=\tilde{p} T+c p T \leq\left(\frac{1}{2}+c p\right) T<p(1-c) T=$ $t$, by (1). So $\operatorname{Prob}\{\tilde{z} \geq t\}$ is negligible.

Zero-Knowledge (If the prover is honest then it is possible to simulate the joint-view of all the verifiers.) This is straightforward, since the number of queries is polynomially bounded. Indeed, $2^{n}<u \leq|N|^{b}=\Theta\left(|x|^{b}\right)$.

\section{Remark}

The number of users $v$ of the broadcast proof can be as much as $n b^{-1}=$ $\frac{1}{8}|x|^{\frac{1}{4}} \log |x|$. We can get $v$ to be linear in $|x|$ if we take the number of rounds to be $t=c(|x| / \log |x|)^{4}, c$ a constant.

\section{A broadcast proof based on the constant round proof of Bellare-Micali-Ostrovsky}

\subsection{Introduction}

Recently Bellare, Micali and Ostrovsky proposed a 5 step perfect zero-knowledge proof for graph isomorphism [2]. Their protocol can be used to design a sabotage-free broadcast proof. Below we present a variation of this protocol. This is a 5 step zero-know- ledge proof for quadratic residuocity ${ }^{2}$. The common input is $x=(I ; N)$, where $I s^{2} \equiv 1(\bmod N)$ and $N$ is the product of two large primes. We define $I_{i} \equiv I(\bmod N)$ and $s_{i} \equiv s(\bmod N), i=1, \ldots, k$. Here $k=|x|$.

\footnotetext{
${ }^{2}$ Bellare-Micali-Ostrowski [2] mention that they have a 5 step perfect zero knowledge interactive proof for quadratic residuocity, but do not describe their protocol. We presume that it is similar to the one given here.
} 


\subsection{A 5 step zero-knowledge interactive proof for qua- dratic residuocity}

Step $1 P$ sends $V$ the number $\chi \equiv \rho^{2}(\bmod N)$ where $\rho \in_{R} Z_{N}^{*}$.

Step $2 V$ sends $P$ the numbers $\beta_{i} \equiv \mu_{i}^{2} \chi^{q_{i}}(\bmod N)$ where $\mu_{i} \in_{R} Z_{N}^{*}$ and $q_{i} \in_{R}\{0,1\}, i=1, \ldots, k$.

Step $3 P$ sends $V$ the numbers $z_{i} \equiv r_{i}^{2}(\bmod N)$ where $r_{i} \in_{R} Z_{N}^{*}, i=$ $1, \ldots, k$.

Step $4 V$ sends $P$ the bits $q_{i}$ and the numbers $\mu_{i}$ for $i=1, \ldots, k$.

Step $5 P$ checks that the $q_{i}$ are bits, that $\mu_{i} \in Z_{N}^{*}$, and that $\beta_{i} \equiv \mu_{i}^{2} \chi^{q_{i}}$ $(\bmod N)$ for $i=1, \ldots, k$. The protocol is halted if this is not so. Otherwise $P$ sends $V$ the numbers $\rho$ and $y_{i} \equiv r_{i} s_{i}^{q_{i}}(\bmod N)$, for $i=1, \ldots, k$.

Step 5' $V$ checks that the $\rho$ and $y_{i}$ belong to $Z_{N}^{*}$, that $\chi \equiv \rho^{2}(\bmod N)$, and that $z_{i} \equiv y_{i}^{2} I_{i}^{q_{i}}(\bmod N)$ for all $i=1, \ldots, k$. If this is the case he accepts, otherwise he rejects. ( $V$ did not sent).

\subsection{A 5 step zero-knowledge sabotage-free broadcast proof for quadratic residuocity}

The broadcast proof is essentially a parallelized version of the Fiat-Shamir scheme, but its novelty is that it is zero-knowledge in 5 rounds. (In the final paper we will present an adjusted version for which there is a substantial saving in broadcast bits.) Below we use the notation $g(|x|) \prec h(|x|)$ to indicate that the functions $g, h$ of $|x|$ are bounded by $\lim _{|x| \rightarrow \infty} g(|x|) / h(|x|)=0$.

Let $h$ be such that $\log |x| \prec h \prec|x| / \log |x|$ and let $n=k / h$. (The case when $h$ is not a factor of $|x|$ can be easily accomodated by taking $k=\Theta(|x|)$; we shall do this in the final paper.) We partition the secrets into subsets

$$
\left(s_{11}, \ldots, s_{1 h}\right), \ldots,\left(s_{i 1}, \ldots, s_{i h}\right), \ldots,\left(s_{n 1}, \ldots, s_{n h}\right) .
$$

Each subset is assigned to an incoming phone line. So the number of queries per line, $q=2^{h}$, is superpolynomial. Then the probability with 
which a dishonest prover can "guess" the correct answer, $1 / q$, is negligible. The public numbers are

$$
\left(I_{11}, \ldots, I_{1 h}\right), \ldots,\left(I_{i 1}, \ldots, I_{i h}\right), \ldots,\left(I_{n 1}, \ldots, I_{n h}\right) .
$$

For the broadcast protocol we use a "first-come, first-serve" network. In this case the lines are exclusive and the verifiers know when their call is selected. In what follows we only consider verifiers which know that they have been selected. So our network model is different from the one we used earlier. We assume that if a verifier's call is selected in Step 2, then the line is reserved for the remaining 3 steps. The protocol has as follows:

Step $1 P$ sends all the $V_{i}, i=1, \ldots, v$, the number $\chi \equiv \rho^{2}(\bmod N)$ where $\rho \in_{R} Z_{N}^{*}$

Step 2 Each $V_{i}$ sends: $W$ the line-number $J_{i} \in_{R}\{1, \ldots, n\}$, and $P$ (via W) $\beta_{J_{i} 1}, \ldots, \beta_{J_{i} n}(\bmod N)$, where $\beta_{J_{i} m} \equiv \mu_{J_{i} m}^{2} \chi^{q_{J_{i} m}}(\bmod N)$ with $\mu_{J_{i} m} \in_{R} Z_{N}^{*}$ and $q_{J_{i} m} \in_{R}\{0,1\}, m=1, \ldots, h$.

Step 3 For each line $J \in\{1, \ldots, n\}, P$ selects a verifier $V_{l} \in_{R}\left\{V_{j} \mid V_{j}\right.$ has selected line $J\}$ and broadcasts all these $l$. Then $P$ broadcasts the numbers $z_{i} \equiv r_{i}^{2}(\bmod N)$ where $r_{i} \in_{R} Z_{N}^{*}, i=11, \ldots, n h$.

Step 4 Each acknowledged $V_{l}$ sends $P$ the bits $q_{J_{l} 1}, \ldots, q_{J_{l} h}$ and the numbers $\mu_{J_{l} 1}, \ldots, \mu_{J_{l} h}$.

Step $5 P$ checks that the $q_{j}$ are bits, that $\mu_{j} \in Z_{N}^{*}$ and that $\beta_{j} \equiv \mu_{j}^{2} \chi^{q_{j}}$ $(\bmod N), j=11, \ldots, n h . P$ broadcasts $\rho$ and $Y_{j}$ where: $Y_{J_{i} 1}=$ $0, \ldots, Y_{J_{i} h}=0$ if any one of these checks has failed for $j=J_{i} m$; otherwise $Y_{j} \equiv r_{j} s_{j}^{q_{j}}(\bmod N)$.

Step 5' Each acknowledged $V_{i}$ checks that the $\rho$ and $Y_{J_{i} m}$ belong to $Z_{N}^{*}$, that $\chi \equiv \rho^{2}(\bmod N)$, and that $z_{J_{i} m} \equiv Y_{J_{i} m}^{2} \cdot I_{J_{i} m}^{q_{J_{i} m}}(\bmod N), m=1, \ldots h$. If this is the case he accepts, otherwise he rejects.

Theorem 2 The protocol described above defines a 1-call sabotage-free broadcast zero-knowledge proof $\left(P, W, V_{1}, \ldots, V_{v}\right)$ for quadratic residuocity.

Proof. Completeness (The honest prover will answer correctly, with overwhelming probability, the first call of every verifier, even when all the 
other verifiers cheat.) This follows directly from the completeness of the single verifier protocol.

Soundness (The probability that a dishonest prover will answer correctly a single call of any honest verifier is negligible.) This follows from the fact that the number of queries per line is superpolynomial. Consequently a dishonest prover can only guess the answer with a negligible probability of success.

Zero-Knowledge (If the prover is honest then it is possible to simulate the joint-view of all the verifiers.) This follows immediately from the fact that the single-verifier proof is zero-knowledge.

\section{Conclusion}

Broadcast protocols are more efficient and have lower bandwidth requirements than repeated single-verifier proofs. (In the final paper we will discuss the bandwidth needed for these proofs.) We have assumed throughout that the network is honest (the telephone companies should be pleased!).

\section{Acknowledgement}

We acknowledge G. Beaumont for general discussions about probability theory and for mentioning Okamoto's bound.

\section{REFERENCES}

[1] L. M. Adleman and K. S. McCurley. Open problems in number theoretic complexity. In D. Johnson, T. Nishizeki, A. Nozaki, and H. Wilf, editors, Discrete Algorithms and Complexity, Proceedings of the Japan-US Joint Seminar (Perspective in Computing series, Vol. 15), pp. 263-286. Academic Press Inc., Orlando, Florida, June 4-6, Kyoto, Japan 1986.

[2] M. Bellare, S. Micali, and R. Ostrovsky. Perfect zero-knowledge in constant rounds. In Proceedings of the twenty second annual ACM Symp. Theory of Computing, STOC, pp. 482-493, May 14-16, 1990. 
[3] M. Ben-Or, S. Goldwasser, J. Kilian, and A. Wigderson. Multi-prover interactive proofs: How to remove intractability assumptions. In Proceedings of the twentieth annual ACM Symp. Theory of Computing, STOC, pp. 113-131, May 2-4, 1988.

[4] M. Blum, A. De Santis, S. Micali, and G. Persiano. Non-interactive zero-knowledge, December 20, 1989.

[5] A. De Santis, S. Micali, and G. Persiano. Non-interactive zeroknowledge with preprocessing. In S. Goldwasser, editor, Advances in Cryptology - Crypto '88, Proceedings (Lecture Notes in Computer Science 403), pp. 269-282. Springer-Verlag, 1990.

[6] Y. Desmedt and M. Yung. Arbitrated unconditionally secure authentication can be unconditionally protected against arbiter's attacks. Presented at Crypto '90, August 12-15, 1990, Santa Barbara, California, U.S.A., to appear in: Advances in Cryptology, Proc. of Crypto '90 (Lecture Notes in Computer Science), Springer-Verlag.

[7] A. Fiat. Broadcast encryption issues. Presented at the rump session of Crypto '90, August 12-15, 1990, Santa Barbara, California, U.S.A., 1990.

[8] A. Fiat and A. Shamir. How to prove yourself: Practical solutions to identification and signature problems. In A. Odlyzko, editor, Advances in Cryptology, Proc. of Crypto'86 (Lecture Notes in Computer Science 263), pp. 186-194. Springer-Verlag, 1987.

[9] S. Goldwasser, S. Micali, and C. Rackoff. The knowledge complexity of interactive proof systems. Siam J. Comput., 18(1), pp. 186-208, February 1989 .

[10] L.C. Guillou and J.-J. Quisquater. A practical zero-knowledge protocol fitted to security micru processor minimizing both transmission and 
memory. In C. G. Günther, editor, Advances in Cryptology, Proc. of Eurocrypt '88 (Lecture Notes in Computer Science 330), Springer-Verlag (1988), pp. 123-128.

[11] N. L. Johnson and S. Kotz. Discrete distributions. John Wiley, New York, 1969.

[12] K. Kurosawa and S. Tsujii. Multi-language zero-knowledge interactive proof system. Presented at Crypto '90, August 12-15, 1990, Santa Barbara, California, U.S.A., to appear in: Advances in Cryptology, Proc. of Crypto '90 (Lecture Notes in Computer Science), Springer-Verlag. 